\section{Tratamiento conservador de ameloblastoma uniquístico. Presentación de un caso}

\section{Conservative treatment of unicystic ameloblastoma. Case presentation}

\section{Resumen}

El ameloblastoma es la segunda neoplasia odontogénica más común, es benigno, presenta un comportamiento agresivo e infiltrante localmente, y con una alta tasa de recidiva; por lo que su tratamiento es muy controversial. Se presenta el caso de un paciente masculino de 14 ańos de edad, sin antecedentes médicos de relevancia, con aumento de volumen en la región mandibular posterior lado izquierda, dura, con color similar a piel adyacente y sin dolor a la palpación. Se le realizó punción y una biopsia incisional, obteniendose como resultado histopatológico: ameloblastoma uniquístico mural, el cual es tratado de forma conservadora con curetaje y ostectomía periférica, evolucionando favorablemente y rehabilitado con implantes osteointegrados. Las modalidades de tratamiento se pueden dividir en radical y conservadora. La técnica a elegir depende principalmente del tipo del ameloblastoma, seguido por la histología, su localización, el tamaño y la edad del paciente; por lo que cada tratamiento debe ser estudiado e individualizado.

Palabras clave: Ameloblastoma; Tratamiento conservador; Implantes dentales (fuente: DeCS BIREME).

\begin{abstract}
Ameloblastomas are the second most common odontogenic neoplasms, they are benign, however, they present an aggressive and locally infiltrative behavior, with a high recurrence rate; so its treatment is very controversial. We present the case of a 14-year-old male patient, with no relevant medical history, with increased volume in the posterior mandibular region left side, hard, with a color like the adjacent skin and painless on palpation. A puncture and an incisional biopsy were performed, obtaining as a histopathological result: mural unicystic ameloblastoma, which was treated conservatively with curettage and peripheral ostectomy, evolving favorably and rehabilitated with osseointegrated implants. Treatment modalities can be divided into radical and conservative. The technique to choose depends mainly on the type of ameloblastoma, followed by histology, its location, size and age of the patient; so each treatment must be studied and individualized.
\end{abstract}

Keywords: Ameoloblastoma; Conservative treatment; Dental implants (source: $\mathrm{MeSH}$ NLM).

\section{Caso Clínico}

Ilan Vinitzky-Brener 1,2,a,b, Tessie Harfuch-Capdevila ${ }^{1,2, a,}$, Carlos Abascal-Quintana ${ }^{3, c}$, Beatriz Aldape-Barrios ${ }^{4, a, d}$

${ }^{1}$ Universidad Anáhuac, México Norte, Facultad de Odontología, Estado de México, México.

2 Instituto Nacional de Enfermedades Respiratorias,

Ciudad de México, México.

${ }^{3}$ Universidad Anáhuac, México Norte, Facultad de Medicina, Estado de México, México.

${ }^{4}$ Universidad Nacional Autónoma de México, Facultad de

Odontología, Ciudad de México, México.

a Cirujano Dentista.

${ }^{\mathrm{b}}$ Especialista en Cirugía Oral y Maxilofacial.

${ }^{c}$ Médico Cirujano. Diplomado en Diagnóstico Clínico en

Patología Bucal.

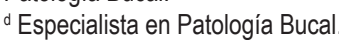

\section{Correspondencia:}

Ilan Vinitzky-Brener: ilanvinitzky@hotmail.com Av. Ejército Nacional No. 1112 int 1001. Polanco. Ciudad de México. México

ORCID: 0000-0001-8378-1444

\section{Coautores:}

Tessie Harfuch-Capdevila: tessie.harfuch@gmail.com ORCID: 0000-0003-4187-2035

Carlos Abascal-Quintana: abascalq@gmail.com ORCID: 0000-0002-2559-9414

Beatriz Aldape-Barrios: patobu1@hotmail.com ORCID: 0000-0001-6468-7815

\section{Editor:}

Eliberto Ruiz Ramirez

Universidad Nacional Mayor de San Marcos, Perú.

Conflicto de intereses: los autores declaran no tener conflictos de interés.

Fuente de financiamiento: autofinanciado.

Recibido: $10 / 06 / 21$

Aceptado: $28 / 10 / 21$

Publicado: 21/01/22

(c) Los autores. Este artículo es publicado por la revista Odontología Sanmarquina de la Facultad de Odontología, Universidad Nacional Mayor de San Marcos. Este es un artículo de acceso abierto, distribuido bajo los términos de la licencia Creative Commons Atribución 4.0 Internacional (CC BY 4.0) [https://creativecommons.org/ licenses/by/4.0/deed.es] que permite el uso, distribución y reproducción en cualquier medio, siempre que la obra original sea debidamente citada de su fuente original. 


\section{Introducción}

El ameloblastoma representa entre el 1\% al 11\% de las neoplasias odontogénicas ${ }^{1}$. Es la segunda en frecuencia después del odontoma ${ }^{2}$. Se le considera un tumor benigno, con comportamiento agresivo e infiltrante localmente, con una alta frecuencia de recidiva ${ }^{3}$. Existe una contraparte maligna del ameloblastoma conocida como carcinoma ameloblástico, el cual combina características histológicas del ameloblastoma con atipia celular con o sin metástasis ${ }^{4}$.

El ameloblastoma se forma a partir del remanente órgano del esmalte de la lámina dental durante el proceso de odontogénesis, también se ha postulado que puede surgir a partir de un quiste dentígero, lo cual es controversial $^{1,3,5}$, o de la capa epitelial basal de las células de la mucosa oral superficial ${ }^{1,3}$. Algunos autores reportan que factores ambientales e individuales también juegan un papel para su desarrollo ${ }^{2}$, así como factores locales asociados, como lo son: irritación (extracción), caries, trauma, infección/inflamación, erupción dental, deficiencias nutricionales o virus ${ }^{6}$. Se han estudiado diversos genes implicados en la patogénesis del ameloblastoma, entre los más importantes destacan los genes Enamelin y cbfaL, los cuales regulan la amelogénesis. Estos y otros genes se activan y se inhiben en el proceso de odontogénesis por lo que alteraciones en los mismos tienen importantes repercusiones clínicas ${ }^{7}$.

Recientemente se ha descubierto la implicación de la vía de señalización MAPK como un paso crítico en su patogénesis, además se ha asociado a mutaciones de la vía de Sonic Hedgehog ${ }^{8}$.

En el 2014, tres reportes identificaron mutaciones MAPK recurrentes en los ameloblastomas, como lo son, FGFR2, RAS y BRAF, los cuales se consideran que se encuentran presentes en el 78-88\% de los ameloblastomas. La más común de estas fue la cinasa BRAF V600E, relacionada además con otras neoplasias ${ }^{8}$.

En 2017 la Organización Mundial de la Salud clasificó los ameloblastomas en cuatro subtipos ${ }^{9}$ :

- Sólidos/Multiquísticos.

- Uniquísticos.

- Extraóseos/ Periférico.

\section{- Desmoplásicos.}

El ameloblastoma uniquístico (AU) fue descrito por primera vez en 1977 por Robinson y Martinez ${ }^{10,11}$. Se presenta como una variante de ameloblastoma que tiene características radiológicas, clínicas y comportamiento de quiste odontogénico ${ }^{10,12}$; sin embargo en la histopatología se observa un epitelio de estirpe ameloblástico ${ }^{11,12}$.

EL AU se presenta principalmente en pacientes jóvenes, entre la primera y segunda década de la vida ${ }^{11,13}$. Tiene una media de edad de presentación a los 16 años y no tiene predilección por género ${ }^{13}$. Clínicamente es asintomático y presenta expansión de corticales, asimetría facial, desplazamiento dental, movilidad dental, resorción radicular, interferencia oclusal y extrusión dental ${ }^{12}$. En la imagen panorámica aparece como una imagen radiolúcida, unilocular, bien delimitada y puede ser confundido con un quiste odontogénico ${ }^{11}$. Alrededor del 90\% se encuentran en la porción posterior de la rama mandibular ${ }^{12}$. En una relación mandíbula : maxilar de 13:1 ${ }^{11}$.

Existen tres subtipos histológicos según Ackerman ${ }^{11}$ :

- Luminal (tipo 1) en el cual el tumor está confinado al epitelio del quiste.

- Intraluminal (tipo 2) el tumor es proyectado hacia el lumen.

- Mural (tipo 3) en el cual el tumor invade la pared de tejido conectivo del quiste ${ }^{11}$, por lo que tiene alta recidiva.

El diagnóstico de AU se hace por las características clínicas y de imagen, siendo necesaria su confirmación ${ }^{11}$, la cual debe ser sometida a múltiples cortes para excluir la posibilidad de ameloblastoma mural en el recubrimiento del quiste ${ }^{1}$.

Los diagnósticos diferenciales para considerar son: quiste dentígero, queratoquiste odontogénico, quiste residual, fibroma ameloblástico, lesión central de células gigantes, tumor odontogénico adenomatoide (inmaduro) entre otras ${ }^{11}$.

Se han descrito diferentes alternativas para el tratamiento del ameloblastoma uniquístico, desde conservadoras hasta radicales, independientemente de la técnica que el cirujano decida, el seguimiento a largo plazo es esencial 12. Se ha reportado una recidiva de hasta el $90 \%$ si no se extirpa completamente ${ }^{13}$, con un intervalo de recurrencia promedio de 7 años ${ }^{10}$.

\section{Reporte del caso}

Se trata de un paciente masculino de 14 ańos de edad, sin antecedentes médicos de relevancia para su padecimiento actual; el cual refiere que aproximadamente 1 semana previa a la consulta nota asimetría facial a expensas de aumento de volumen en región mandibular izquierda, por lo que acude con un odontólogo general, el cual solicita ortopantomografía y lo refiere al especialista en cirugía oral y maxilofacial para valoración $\mathrm{y}$ tratamiento. Al interrogatorio inicial refieren paciente y familiares, haberse percatado hace algunos días del aumento de volumen, no refiere dolor, parestesia u otro dato asociado. A la exploración física se observa aumento de volumen en región mandibular posterior lado izquierdo, duro, con color similar a piel adyacente y sin dolor a la palpación. Cuello sin alteraciones, adecuada apertura oral, intra oralmente presenta aumento de volumen en región mandibular posterior lado izquierdo desde zona de premolares hasta zona retro molar con expansión de corticales, con zona ulcerada en superficie oclusal de región de segundo y tercer molar (Figura 1). En ortopantomografía se observa zona radiolúcida bien delimitada multilocular que abarca desde primer premolar inferior izquierdo hasta rama mandibular ipsilateral con tercer molar incluido en la 
lesión y rizolisis de primero y segundo molar (Figura 2). Se solicita tomografía cone beam con cortes axiales, coronales y reconstrucción $3 \mathrm{~d}$, observando zona hipo densa en región posterior de cuerpo y rama mandibular lado izquierdo, con expansión de corticales (Figura 3). Se procede a realizar punción y biopsia incisional, obteniendo como resultado histopatológico: ameloblastoma uniquístico mural. Se procede a realizar curetaje de la lesión, ostectomía periférica y extracción de órganos dentarios involucrados (segundo premolar, primero, segundo y tercer molar), bajo anestesia general (Figura 4 y 5). El manejo farmacológico postoperatorio se realizó con clindamicina $300 \mathrm{mg}$ cada 6 horas por 7 días, ibuprofeno $600 \mathrm{mg}$ cada 8 horas por 5 días, ketorolaco $30 \mathrm{mg}$ en caso de dolor agudo y colutorios con gluconato de clorhexidina $0,12 \%$ cada 8 horas por 14 días. Se dan indicaciones generales al paciente, incluyendo dieta blanda estricta por 4 semanas por riesgo de fractura mandibular. El postoperatorio cursa sin complicaciones. Se envía para estudio histopatológico confirmando el resultado de ameloblastoma uniquístico (Figura 6). El paciente se mantiene en control clínico y radiográfico continuo. En el control a 5 ańos el paciente se encuentra asintomático, clínicamente sin aumento de volumen, con simetría facial, adecuado proceso de cicatrización de la mucosa. En los estudios de imagen, se observa adecuada regeneración del hueso mandibular sin datos de recidiva de la lesión (Figuras 7 y 8). Basándonos en la adecuada respuesta al tratamiento se ofrece al paciente la colocación de implantes dentales para reemplazar los dientes perdidos y mejorar la función masticatoria, así como la estética. Se realiza estudio tomográfico, planeación digital y elaboración de guía quirúrgica digital (ya que los implantes dentales requerían una angulación precisa que logrará evitar lesionar el nervio dentario inferior). Se colocan tres implantes dentales ( ${ }^{\oplus}$ Hiossen), los cuales se mantienen sin carga por 6 meses y tras verificar su adecuada osteointegración son rehabilitados con coronas monolíticas (Figuras 9, 10 y 11).

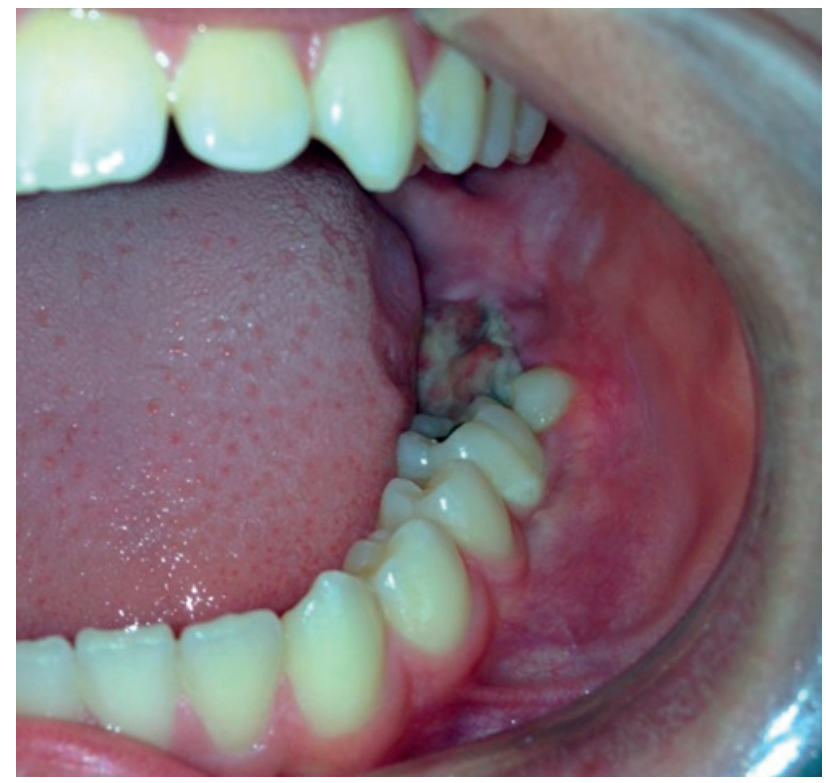

Figura 1. Aspecto Intraoral de la lesión

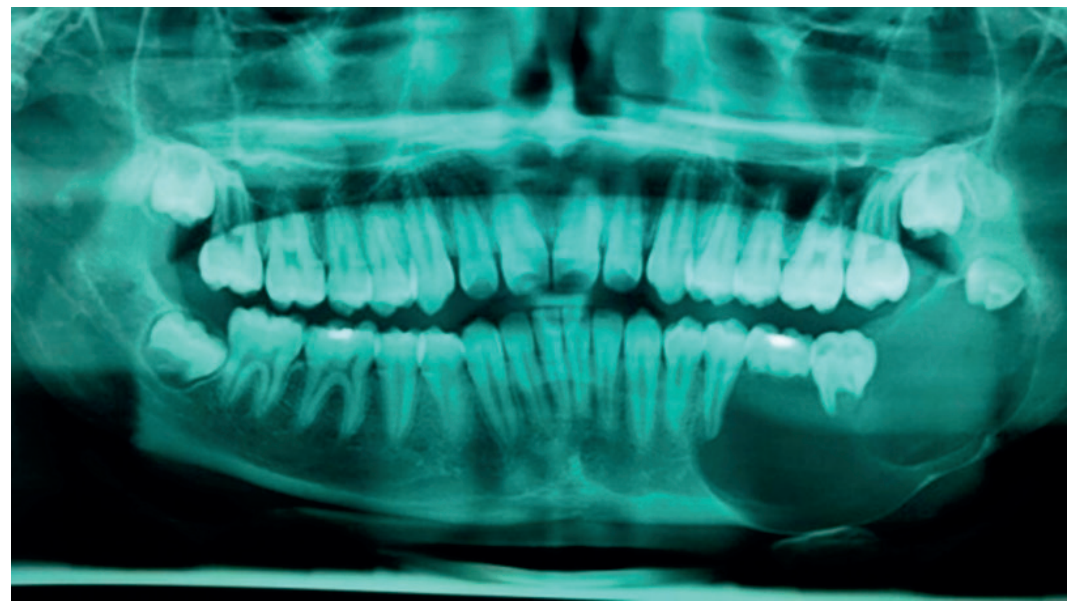

Figura 2. Ortopantomografía Inicial 


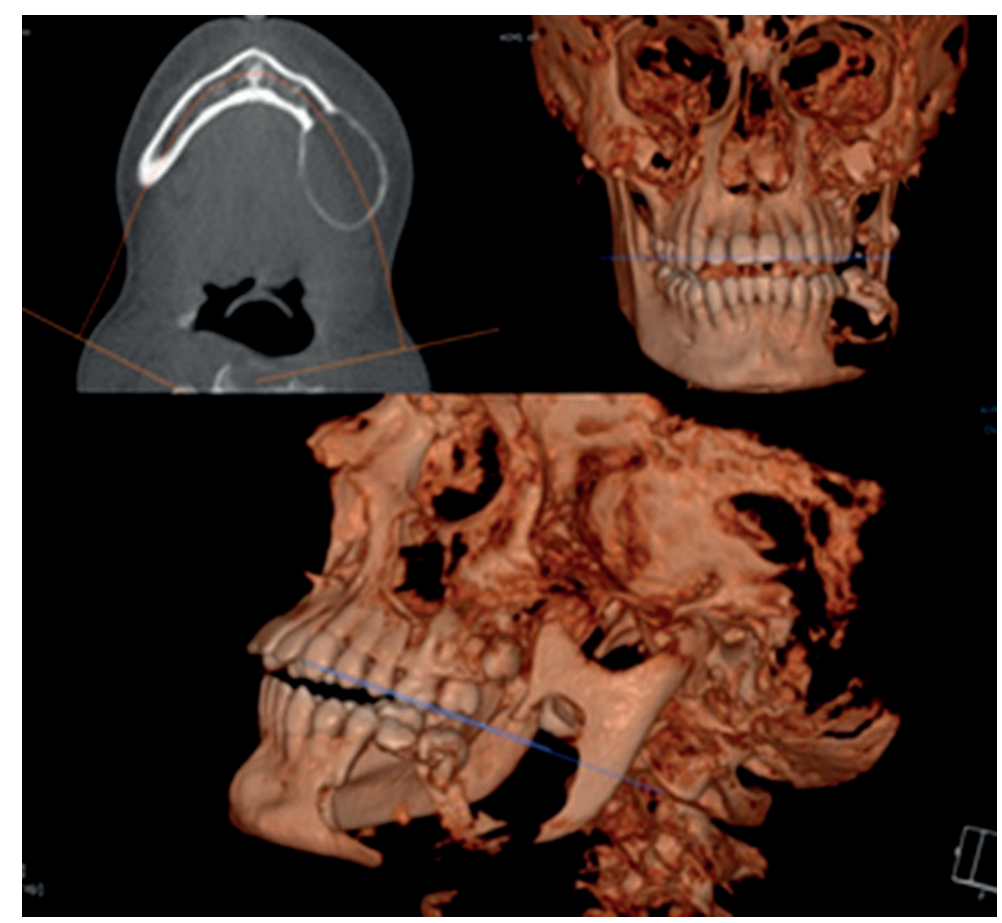

Figura 3. Tomografía cone beam inicial

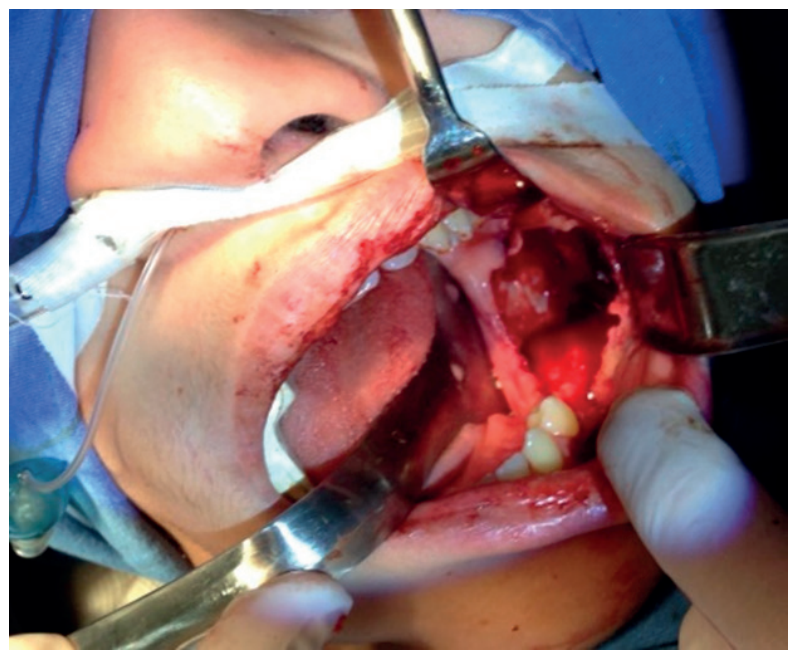

Figura 4. Aspecto transoperatorio del lecho quirúrgico

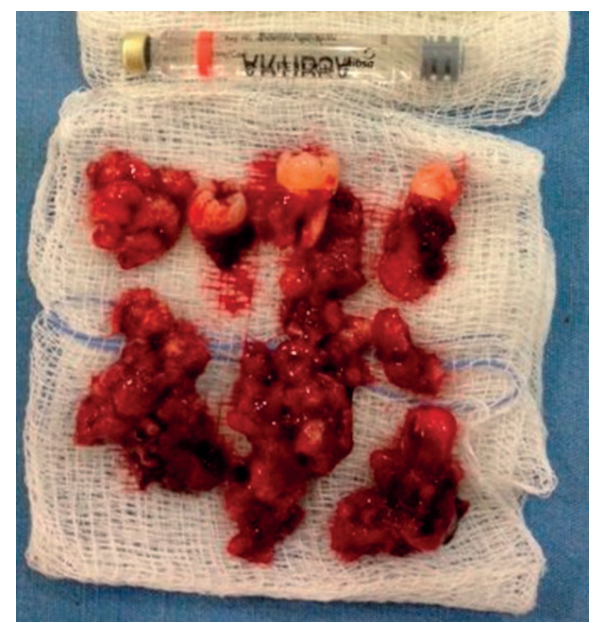

Figura 5. Aspecto macroscópico de la lesión y dientes extraidos 


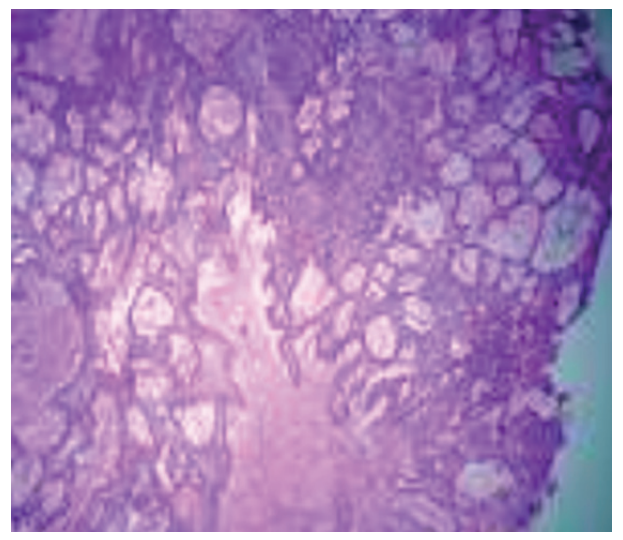

Figura 6. Corte histológico teñido con HyE

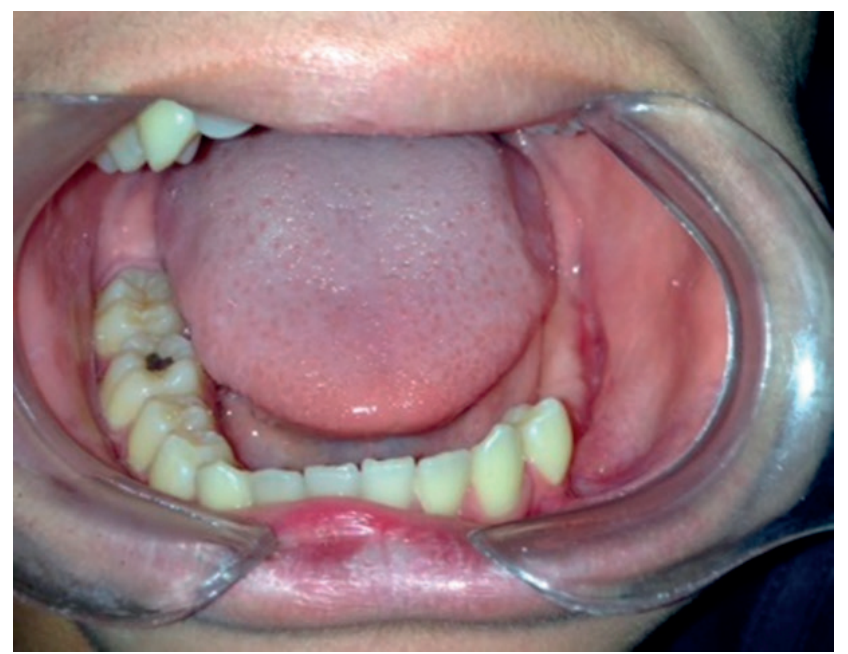

Figura 7. Control a 5 años imagen clínica

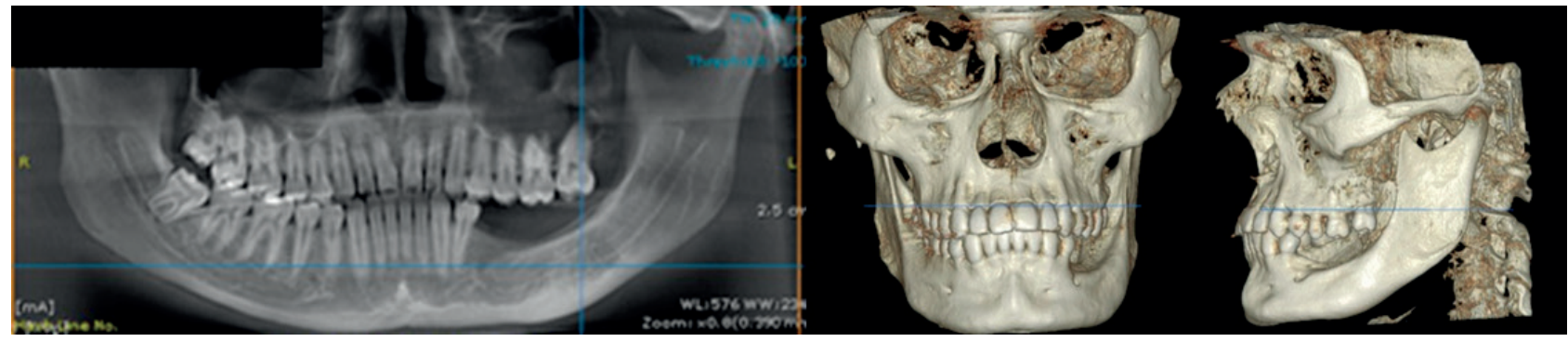

Figura 8. Control a 5 años, estudios de imagen

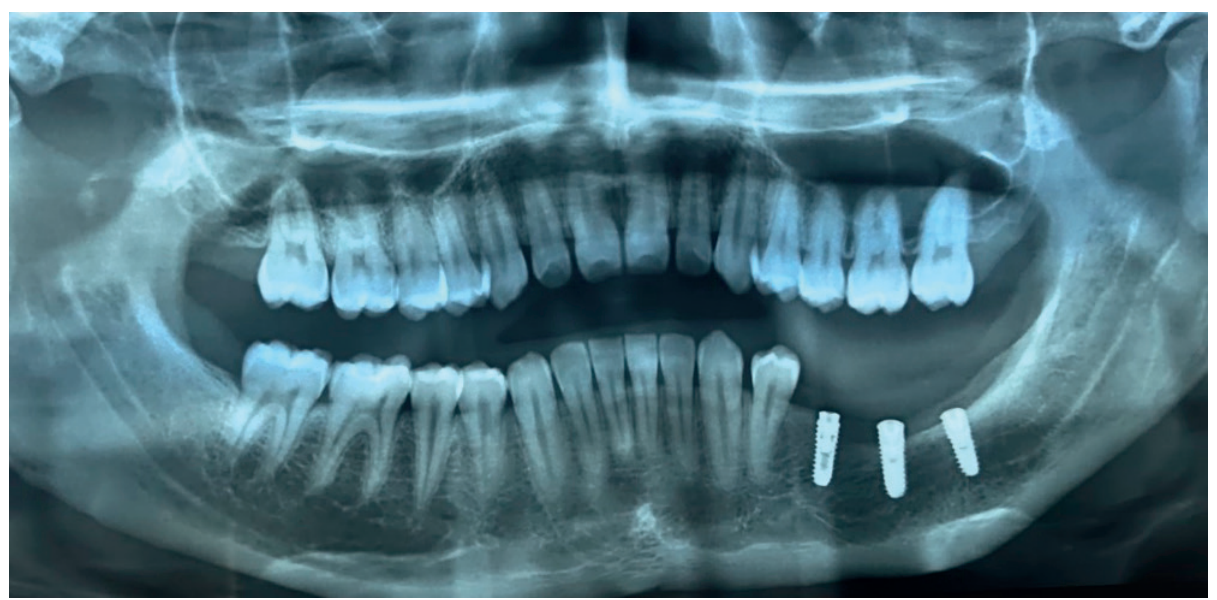

Figura 9. Rehabilitación con implantes dentales en zona de órganos dentales 35, 36 y 37; 5 años después 


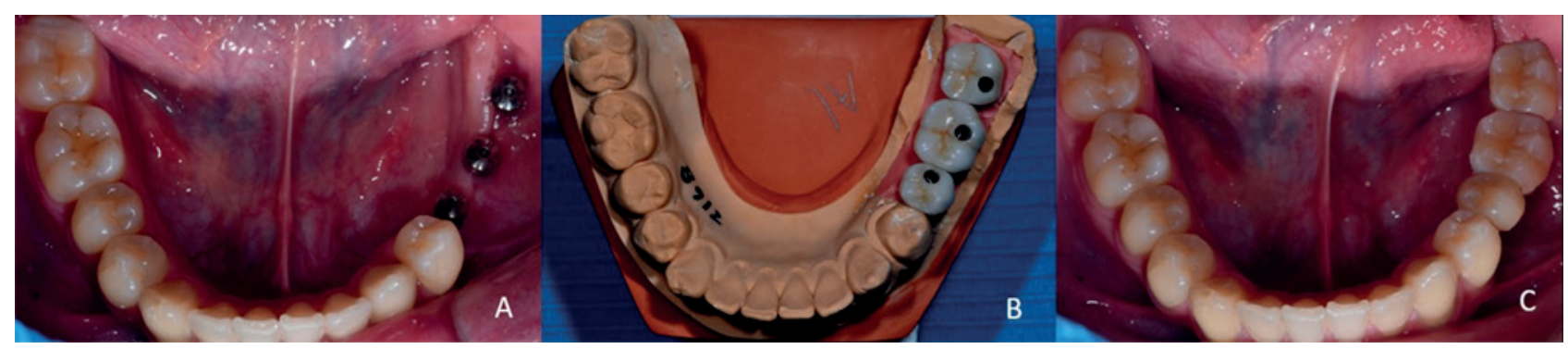

Figura 10. Fase protésica: A. Tornillos de cicatrización. B. Prótesis sobre modelo de trabajo. C. Rehabilitación final (Fase protésica Dr Jorge López Casas)

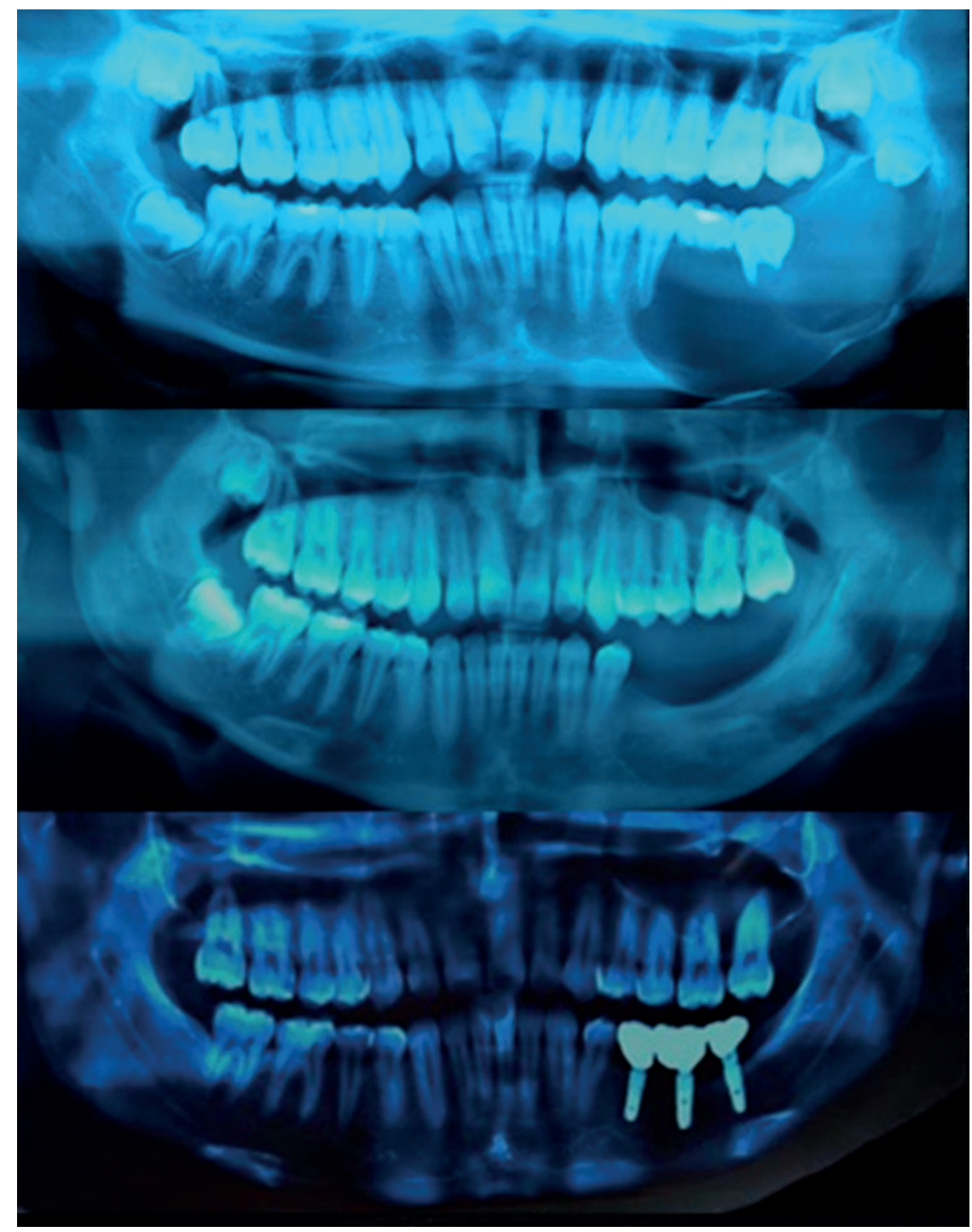

Figura 11. Ortopantomografías preoperatorias, postoperatorias y rehabilitación completa

Actualmente el paciente se encuentra asintomático con adecuada estética, función masticatoria y sin alteraciones sensoriales. Continuará en control y seguimiento a largo plazo.

\section{Discusión}

A pesar de ser una neoplasia benigna, su comportamiento agresivo e infiltrante localmente, plantea una problemática para su tratamiento ${ }^{3}$. Las modalidades pueden dividirse en conservadora, siendo las opciones: enucleación, curetaje y marzupialización, con o sin terapia adyuvante ${ }^{5}$; y cirugía radical, realizando una resección en bloque ${ }^{1,7}$. Además de valorar el tipo clínico, es necesario conocer la histopatología del tumor por medio de una biopsia incisional previa al tratamiento definitivo; así como la localización, el tamańo y la edad del paciente, es decir el tratamiento debe ser individualizado ${ }^{10}$. Para el tipo Ackerman tipo 1 o 2 es posible realizar un 
tratamiento conservador, por medio de enucleación ${ }^{11}$, y para el tipo Ackerman tipo 3 se considera que debe ser tratado radicalmente por su alta recurrencia, siendo esta aproximadamente del $30 \%$ si no se extirpa completamente ${ }^{11,12}$. Es difícil diferenciar las variantes luminal e intraluminal preoperatoriamente, ya que puede observarse distinto en las diferentes zonas del tumor, por lo que se podría errar en el tratamiento adecuado ${ }^{5}$, siendo necesario el estudio histopatológico para su correcto abordaje ${ }^{11}$. El objetivo del tratamiento incluye una excisión completa del tumor con márgenes lineales, confirmados histológicamente postoperatorio o por imagen intraoperatoria, la restauración de la función y reconstrucción ósea temprana ${ }^{5}$.

Existen nuevos estudios que permiten lograr un margen intraoperatorio satisfactorio de tejido sano, como lo es la tomografía computarizada con detectores planos FDCT (del inglés, flat panel detector CT) ${ }^{5,14}$. Se ha observado que los ameloblastomas tienen una alta recurrencia cuando se realiza cirugía de manera conservadora ${ }^{1,2}$; $\sin$ embargo, en la cirugía radical se asocia a complicaciones como posible deformidad facial y una significativa morbilidad ${ }^{8}$.

La terapia coadyuvante ha aumentado la eficacia de este tipo de tratamientos siendo, la crioterapia, electrocauterio y la solución de Carnoy ${ }^{11}$. La crioterapia disminuye la recurrencia pero es asociada a fracturas en zona de patología y dehiscencia de la herida, con resultados similares para las otras técnicas. No son apropiadas donde el tejido blando y la cortical se encuentren adelgazadas o existan perforaciones ${ }^{11}$.

El tratamiento radical mediante la resección en bloque es un abordaje que asegura la máxima eliminación de los ameloblastomas sólido/ multiquístico, así como la variante intramural del uniquístico ${ }^{11}$, disminuyendo la tasa de recurrencia $(0-10 \%)$. Se recomienda la realización de márgenes quirúrgicos de por lo menos $1 \mathrm{~cm}$ para una terapia satisfactoria, ya que en la literatura se ha sugerido que el ameloblastoma se puede extender de 2,3 a $8 \mathrm{~mm}$ dentro del hueso esponjoso ${ }^{5}$.

La radioterapia y quimioterapia no son efectivas en el manejo, ya que están asociadas con una tasa de falla del $100 \%$ y complicaciones secundarias serias, como osteomielitis, que llevan al desarrollo de sarcoma o a la muerte ${ }^{5}$. Actualmente se encuentra en investigación el tratamiento farmacológico de las neoplasias con mutaciones de la vía MAPK, aunque otras mutaciones también pueden ser blanco de terapia, las cuales pueden ser útiles en los casos metastásicos y localmente agresivos que no son candidatos a cirugía ${ }^{8}$. Entre estos tratamientos moleculares que se están investigando para el tratamiento no quirúrgico del ameloblastoma se proponen inhibidores de la vía de sonic hedgehog, en los que se incluyen la cyclopamina, robotnikinin, KAAD-cyclopamina, Jervine, IPI926, GDC-0449, biarylcarboxamida, CUR61414, SANT1, SANT2, SANT3, SANT4, JK184, y GANT61 ${ }^{15}$.
Hasta el momento no hay un consenso en la literatura sobre el tratamiento ideal para los ameloblastomas uniquísticos y diferentes autores han expresado lo que ellos consideran es el tratamiento ideal para estas lesiones. Gardner ${ }^{16}$ señala que existe una diferencia en el comportamiento patológico entre aquellas lesiones que son simplemente quísticas o muestran proliferación intraluminal (tipos 1 y 2 ) y aquellas en las que el epitelio penetra y rompe la pared fibrosa invadiendo el hueso esponjoso (tipo 3). Hay que tener en cuenta que los tipos 1 y 2 favorecieron un resultado exitoso con un tratamiento conservador.

Marx ${ }^{17}$ considera que debe ser tratado por resección con márgenes de seguridad por sus características clínicas e histológicas y cita a Shatkin y Hoffemeister que, en el año 1965 , se refieren al tratamiento conservador como una "ilusión", de la cual se disipa a veces como desfiguración o como muerte del paciente, afirmando que por eso debe ser tratado agresivamente.

Hendra et al. ${ }^{18}$ realizan una revisión sistemática y metaanálisis en el que concluyen que el tratamiento radical es significativamente favorable para todos los ameloblastomas uniquístico, sólido o multiquístico. McClary et al. ${ }^{19}$ recomiendan un margen de 1 a $1,5 \mathrm{~cm}$ para ameloblastomas uniquísticos. Además del tipo de ameloblastoma, se debe considerar diferentes factores que nos llevan a la decisión de realizar un tratamiento conservador o radical, como son, la extensión del tumor, compromiso con estructuras adyacentes, el tipo histológico, la edad del paciente, la salud general del paciente, además de su completa comprensión de la posibilidad de recidivar, su compromiso y cooperación para realizar el seguimiento apropiado.

Debemos tomar en cuenta que, en la gran mayoría de los casos, este tipo de patología se presenta en pacientes jóvenes y así considerar que el tipo de tratamiento que le realicemos va a influir enormemente en la calidad de vida de estos pacientes. El tratamiento conservador juega un rol excelente y es asociado con una recuperación más rápida y eficiente atribuida a la felixibilidad de los huesos jóvenes; es por eso que pueden ser tratados exitosamente con tratamiento conservador, sin afectar su calidad de vida. Meshram et al. ${ }^{20}$ realizan un estudio prospectivo con un grupo de pacientes a los que realizan tratamiento conservador y apoyan completamente este tipo de tratamiento por su baja morbilidad y la capacidad de regeneración que presentan en comparación a la resección.

La elección del tratamiento debe de ser individualizada tomando en cuenta múltiples factores y debe ser discutida ampliamente con el paciente. Aunque es cierto que el tratamiento radical ofrece una tasa mucho más baja de recidiva; se debe considerar que implica una morbilidad importante, la cual conlleva alteraciones estéticas, funcionales, sensoriales y psicológicas que impactan de forma directa en la calidad de vida del paciente. Es por esto que el tratamiento conservador en casos adecuados y con controles estrictos ofrece una excelente alternativa 
para estos pacientes, disminuyendo considerablemente las secuelas de una cirugía extensa y mutilante.

\section{Referencias bibliográficas}

1. Ghandhi D, Ayoub AF, Pogrel MA, MacDonald G, Brocklebank LM, Moos KF. Ameloblastoma: a surgeon's dilemma. J Oral Maxillofac Surg. 2006;64(7):1010-4. DOI: 10.1016/j.joms.2006.03.022.

2. Kessler HP. Intraosseous ameloblastoma. Oral Maxillofac Surg Clin North Am. 2004;16(3):309-22. DOI: 10.1016/j.coms.2004.03.001.

3. Argandońa Pozo J, Espinoza J . Ameloblastoma uniquístico, bases del tratamiento conservador. Presentación de caso clínico y actualización bibliográfica Rev Esp Cir Oral Maxilofac. 2010;32(2):88-91.

4. Licéaga Reyes R, Vinitzky Brener I, Alatorre Pérez S, Mosqueda Taylor A. Carcinoma Ameloblástico. Revisión de la literatura y Presentación de un caso. Rev Mex Cir Bucal Maxilofac. 2011;7(1):15-19.

5. Chae MP, Smoll NR, Hunter-Smith DJ, Rozen WM Establishing the Natural History and Growth Rate of Ameloblastoma with Implications for Management: Systematic Review and Meta-Analysis. PLoS ONE. 2015;10(2):e0117241. DOI:10.1371/journal. pone. 0117241 .

6. Kim SG, Jang HS. Ameloblastoma: a clinical, radiographic, and histopathologic analysis of 71 cases. Oral Surg Oral Med Oral Pathol Oral Radiol Endod. 2001;91(6):649-53. DOI: 10.1067/moe.2001.114160.

7. Nagi R, Sahu S, Rakesh N. Molecular and genetic aspects in the etiopathogenesis of ameloblastoma: An update. J Oral Maxillofac Pathol. 2016;20(3):497-504. DOI: 10.4103/0973-029X.190954.

8. Brown NA, Betz BL. Ameloblastoma: A Review of Recent Molecular Pathogenetic Discoveries. Biomark Cancer. 2015;7(2):19-24. DOI: 10.4137/BIC.S29329.

9. El-Naggar AK, Chan JKC, Grandis JR, Takata T, Slootweg PJ. WHO classification of head and neck tumours. Lyon, France: International Agency for Research on Cancer. 2018; 472(3):311-313. DOI:10.1007/s00428018-2320-6.

10. Torres-Lagares D, Infante-Cossío P, Hernández-Guisado JM, Gutiérrez-Pérez JL. Mandibular ameloblastoma. A review of the literature and presentation of six cases. Med Oral Patol Oral Cir Bucal. 2005;10:231-8. DOI:10.4317/medoral.24104.
11. Kalaskar R, Unawane AS, Kalaskar AR, Pandilwar P. Conservative management of unicystic ameloblastoma in a young child: Report of two cases. Contemp Clin Dent. 2011;2:359-63. DOI:10.4103/0976-237X.91804.

12. Gupta N, Saxena S, Rathod VC, Aggarwal P. Unicystic ameloblastoma of the mandible. J Oral Maxillofac Pathol. 2011; 15: 228-231. DOI: 10.4103/0973-029X.84511.

13. Masthan KM, Anitha N, Krupaa J, Manikkam S. Ameloblastoma. J Pharm Bioallied Sci. 2015;7(1):S167-70. DOI: $10.4103 / 0975-7406.155891$.

14. De Silva I, Rozen WM, Ramakrishnan A, Mirkazemi M, Baillieu C, Ptasznik R, Leong J. Achieving adequate margins in ameloblastoma resection: the role for intra-operative specimen imaging. Clinical report and systematic review. PLoS One. 2012;7(10):e47897. DOI: 10.1371/ journal.pone.0047897.

15. Mishra P, Panda A, Bandyopadhyay A, Kumar H, Mohiddin, G. Sonic Hedgehog Signalling Pathway and Ameloblastoma - A Review. Journal of clinical and diagnostic research: JCDR. 2015;9(11):ZE10-ZE13. DOI: 10.7860/JCDR/2015/15443.6750.

16. Gardner DG. A pathologist's approach to the treatment of ameloblastoma. J Oral Maxillofac Surg. 1984;42:161166. DOI: 10.1016/S0278-2391(84)80026-9.

17. Carlson ER, Marx RE. The Ameloblastoma: Primary, Curative Surgical Management. J. Oral Maxillofac Surg. 2016;64(3):484-494. DOI: 10.1016/j. joms.2005.11.032.

18. Hendra FN, Natsir Kalla DS, Van Cann EM, de Vet HCW, Helder MN, Forouzanfar T. Radical vs conservative treatment of intraosseous ameloblastoma: systematic review and meta-analysis. Oral Dis. 2019;25(7):16831696. DOI:10.1111/odi.13014.

19. McClary AC, West RB, McClary AC, Pollack JR, Fischbein NJ, Holsinger CF, Sunwoo J, Colevas AD, Sirjani D. Ameloblastoma: a clinical review and trends in management. Eur Arch Otorhinolaryngol. 2016;273(7):164961. DOI: $10.1007 / \mathrm{s} 00405-015-3631-8$.

20. Meshram M, Sagarka L, Dhuvad J, Anchlia S, Vyas S, Shah H. Conservative Management of Unicystic Ameloblastoma in Young Patients: A Prospective Single-Center Trial and Review of Literature. Journal of maxillofacial and oral surgery. 2017;16(3):333-341. DOI:10.1007/ s12663-016-0987-2. 